\title{
Quaderni
}

QUADERNI Communication, technologies, pouvoir

79 | Automne 2012

Produire la démocratie

\section{« La participation en kit » : l'horizon funèbre de l'idéal participatif}

Julia Bonaccorsi et Magali Nonjon

\section{(2) OpenEdition}

Édition électronique

URL : http://journals.openedition.org/quaderni/618

DOI : 10.4000/quaderni.618

ISSN : 2105-2956

Éditeur

Les éditions de la Maison des sciences de l'Homme

Édition imprimée

Date de publication : 5 octobre 2012

Pagination : 29-44

Référence électronique

Julia Bonaccorsi et Magali Nonjon, « «La participation en kit » : l'horizon funèbre de l'idéal participatif », Quaderni [En ligne], 79 | Automne 2012, mis en ligne le 05 octobre 2014, consulté le 20 avril 2019.

URL : http://journals.openedition.org/quaderni/618; DOI : 10.4000/quaderni.618 


\section{$D$ ossier}

\section{"La participation en kit" :}

l'horizon funèbre de l'idéal

Une véritable commande publique participative semble s'être progressivement développée, formalisée et codifiée depuis les années 90 attestant en France de l'existence d'une offre institutionnelle de participation ${ }^{1}$. Ainsi, la plupart des dispositifs participatifs contemporains ont été initiés dans le cadre de commandes publiques réglementées. Des fonds ont été attribués à la gestion, l'animation et l'évaluation des instances participatives créant un véritable marché du conseil en participation. Néanmoins, si l'univers de la participation s'est transformé, les recherches sur la démocratie participatif

\section{Julia Bonaccorsi}

Enseignante-chercheuse en Sciences de l'information et de la communication Université Paris Est, CEDITEC

\section{Magali Nonjon}

Enseignante-chercheuse en Science politique Université d'Avignon et des Pays du Vaucluse, LBNC participative semblent encore aujourd'hui se focaliser sur l'analyse des dispositifs en tant que tels, témoignant d'un certain " tropisme procédural $»^{2}$. Les questionnements davantage axés sur la sociologie de la participation, sur les conditions de délibération ou encore sur l'efficacité et les effets des débats publics n'attachent encore que très peu d'importance à la dimension ingénierique de la participation. Pourtant, ce décalage entre les questionnements investis aujourd'hui en sciences sociales sur la démocratie participative et l'évolution des politiques participatives ne résiste pas à l'observation empirique. Les rencontres professionnelles sur les enjeux de participation se sont en effet multipliées sur le territoire célébrant l'impérieuse nécessité pour les promoteurs de la participation de se doter de savoir-faire, d'outils et de méthodes. On pense notamment aux dernières rencontres des professionnels de la participation organisées en novembre 2011 par le conseil général du Val-deMarne révélant tant dans son contenu que dans sa forme, l'intérêt grandissant porté par les acteurs de la participation à l'ingénierie participative ${ }^{3}$. 
Regroupant près de 192 participants, ces rencontres avaient pour principal objectif « de créer un espace d'échanges et de discussions réservés aux professionnels de la démocratie participative " et de "favoriser les échanges d'expériences, la confrontation des méthodes et la mutualisation des savoir-faire $»^{4}$.

Face à de telles évolutions, l'objectif de notre contribution est triple. Tout d'abord, elle fait le pari scientifique de prendre au sérieux ces transformations du milieu de la participation et invite à ce titre à repenser les questionnements à l'aune desquels la démocratie participative est aujourd'hui interrogée. Il s'agit bien pour nous d'étudier la part ingénierique de la participation. Le terme ingénierie nous permet de nous situer dans un juste milieu entre une approche centrée sur les acteurs et une perspective qui ne tiendrait compte que de la dimension fonctionnelle des dispositifs : il y a des ingénieurs de la participation, ceux-ci produisent ou mobilisent des machines / outillages qui doivent être analysés comme des configurations signifiantes. L'approche que nous défendrons dans cet article articule points de vue sociologique et sémiologique sur l'ingénierie participative. Ce double cadre théorique répond à l'ambition de considérer de manière équilibrée ce que nous désignons comme " ingénierie ", c'est-à-dire les logiques des acteurs et les enjeux signifiants des formes matérielles produites, notamment les « kits» (design participatif) 5 .

Deuxièmement, nous souhaiterions montrer que loin d'être neutres et transparents, ces outils nourrissent et reconduisent des standards de la participation. La professionnalisation du secteur en est un des facteurs (démultiplication des prestataires, conception d'outils clés en main, etc.) (Nonjon, 2006), produisant des effets de lissage et de préfiguration des rôles des acteurs de la démocratie participative qu'il convient d'interroger. Derrière l'hétérogénéité des techniques, il semble aujourd'hui se dégager des registres de légitimation, des modalités de présentation, de construction et d'utilisation homogènes. Nous pensons notamment à l'importation de techniques issues du management et de la communication, à l'intérêt porté à la question de la transférabilité des méthodes ${ }^{6}$. Dit autrement, la professionnalisation de la participation aurait favorisé l'émergence d'une participation en « kit ». Or, si nous ne pouvons réduire les effets de la professionnalisation à cette " mise en kit » de la participation, on ne saurait la négliger.

Enfin, nous souhaiterions montrer en quoi ce processus de standardisation de la participation n'est pas sans effet sur ce que signifie « participer» aujourd'hui. Plus précisément, c'est bien la naturalisation des liens entre outils et participation (Bonaccorsi, Julliard, 2010) et l'attention grandissante portée aux stratégies pour garantir le caractère participatif de ces outils qui seront interrogées dans cet article. La participation n'est aujourd'hui plus seulement outillée mais parfois simplement réduite à ses outils, introduisant une véritable confusion entre participation et prestations proposées par les professionnels. 
Professionnalisation de la participation et émergence des « kits » participatifs

\section{Marché de la participation, uniformisation des pratiques et standardisation des outils}

Fréquenté par une nébuleuse d'acteurs aux prestations et aux profils variés (anciens travailleurs sociaux, chefs de projet politique de la ville, architectes, urbanistes, conseillers en management, marketing, consultantschercheurs, etc.), le marché de la participation est au premier abord particulièrement insaisissable (Nonjon, 2006). L'existence de cette nébuleuse participative ne doit pas pour autant laisser croire qu'il n'y a pas eu de dynamique de structuration et de standardisation des profils et des pratiques depuis les années soixante-dix. Sans rentrer dans la complexité des mécanismes de professionnalisation, les professionnels de la participation contribuent autant à façonner la commande publique participative que cette dernière les façonne (Nonjon, 2006). On peut entre autres relever les évolutions suivantes :

- En centrant la participation sur des enjeux de méthodes, les «militants » de la première génération, celle des luttes urbaines, ont contribué à la diffusion d'un savoir-faire participatif ne reposant pas essentiellement sur leurs expériences militantes passées ou présentes, mais davantage sur des compétences intellectuelles (capacité à théoriser leurs pratiques et à les modéliser) et techniques (produire des outils) plus facilement captables par d'autres univers professionnels ${ }^{7}$ ).

- Par ailleurs, les pionniers des luttes urbaines ont érigé la question de la traduction comme enjeu primordial au sein des instances participatives. En se concentrant sur la mise au point de méthodologies pour faire parler les habitants et relayer leurs paroles, ces militants ont ainsi accéléré la concurrence sur les modes de mise en forme et de traduction de la parole habitante. Ce faisant, ils ont favorisé l'arrivée dans le milieu de profils professionnels plus techniques, ne disposant pas forcément de ressources militantes à proprement parler pour accompagner le passage "de l'avis à la formulation de propositions ", révéler la « connaissance sociale 》 et "l'expertise d'usage " des habitants. Les architectes et urbanistes ont pu, par exemple, faire prévaloir leur maitrise du langage de la planification urbaine, leur capacité à traduire des besoins en espaces, à dessiner, à lire des plans. C'est également le cas des professionnels, issus des métiers de la communication et du journalisme, qui ont pu également sans grandes difficultés se positionner sur cet enjeu de traduction, mais également le cas du design industriel et numérique, dont la Fondation Internet Nouvelle Génération (FING) ${ }^{8}$ est certainement la plus symptomatique?.

- Enfin, en réinvestissant les savoirs acquis lors des expériences laboratoires des années 70-80 dans le «process conseil », les militants de la première heure ont légitimé sur le marché l'arrivée de profils issus du conseil en management. Certains consultants en management allant même jusqu'à naturaliser leur position sur le créneau de la participation, «de la satisfaction du client, à celle de l'usager puis celle de l'habitant $»^{10}$.

Dans un contexte de concurrence accrue, les « militants » de la participation de la première génération comme les plus contemporains, 
ont ainsi été contraints de professionnaliser leurs savoir-faire et de s'aligner sur l'offre de service toujours plus gestionnaire proposée par les nouveaux entrants : développement des outils de communication, séquençage des interventions, introduction d'un choix à la carte, création d'événementiels, d'expositions, etc. Les reconversions successives des anciens « militants » comme l'arrivée de nouveaux entrants sur le marché de la participation ont ainsi facilité les transferts et les importations de modèles issus notamment de l'entreprise privée et contribué à un processus d'homogénéisation de l'ingénierie participative. Les prestations proposées pour favoriser la mobilisation et l'expression des citoyens, ont ainsi tendance à se ressembler de plus en plus (et cela malgré les stratégies de démarcation utilisées par les professionnels pour se différencier les uns des autres) : questionnaire, sondage, enquête sociologique, forum, débat, jury de citoyens, exposition itinérante, etc.

Ainsi, sans pour autant postuler que la professionnalisation de la participation peut se résumer à un jeu à somme nulle attestant du repli, voire de la fin des militants sur ce secteur ${ }^{11}$, la captation du marché par de nouveaux entrants, aux profils davantage issus de la communication et du conseil en management depuis les années quatre-vingt-dix, a contribué à une dynamique d'homogénéisation. Cette dynamique d'homogénéisation est bien évidemment largement renforcée par les évolutions parallèles de la commande publique participative dont on ne peut ici détailler les mécanismes : importance grandissante accordée aux activités de conseil, démultiplication des commandes, etc. ${ }^{12}$.

\section{Le séquençage des procédures}

Plusieurs indices témoignent de ce mécanisme d'homogénéisation et de standardisation du milieu de la participation ${ }^{13}$. Parmi ces indices, nous nous focaliserons ici sur l'impératif de séquençage qu'ont imposé la professionnalisation et la marchandisation de la participation. Frisant la caricature, chez certains «professionnels », comme en atteste l'encadré ci-dessous où les séquences du "Service Lab " (application numérique permettant de dresser des 《 cartes de la participation ») sont numérotées, ce procédé est aujourd'hui largement généralisé.

\section{Encadré 1 : « Service Lab Framework : nous vous accompagnons de $\mathrm{A}$ à $\mathrm{Z}$ »}

- En fonction du contexte territorial, des thématiques que vous souhaitez voir traitées par l'étude, nous engageons une Phase d'Imprégnation pendant laquelle une équipe composée de designers, sociologues, urbanistes, se rend sur place afin de découvrir le territoire, rencontrer les acteurs particulièrement concernés.

- Les données récoltées, portraits et interviews réalisés sont agrégés et partagés via un outil en ligne que nous avons spécialement conçu à cet effet : la « Carte des Observation » voir nos outils...

- L'étape suivante, la Phase d'Idéation permet d'entrer de plain-pied dans la démarche de concertation par un biais ludique et valorisant : la co-création, co-génération d'idées de services pour le territoire dans le cadre d'ateliers créatifs qui regroupent tant des élus, acteurs associatifs et économiques locaux que des habitants de tous horizons.

- L'analyse des productions des ateliers permet de sélectionner les pistes les plus convaincantes, qui peuvent alors être détaillées sous la forme de scénarios d'usage dessinés, puis testés et améliorés par itérations successives.

- Enfin, notre équipe vous accompagne dans la médiation 
publique qui présente le/les projets innovants finalement retenus.

- Ceux-ci peuvent alors être réalisés : nous sommes encore là pour vous. La Phase de Transmission et Réalisation nous engage dans la concrétisation d'un cahier des charges, et une assistance dans le suivi du chantier $\mathrm{du} / \mathrm{des}$ nouveaux services.

\section{Encadré 2 et 3 : schématisation du processus (Source : http://www.servicelab.fr/offres/)}
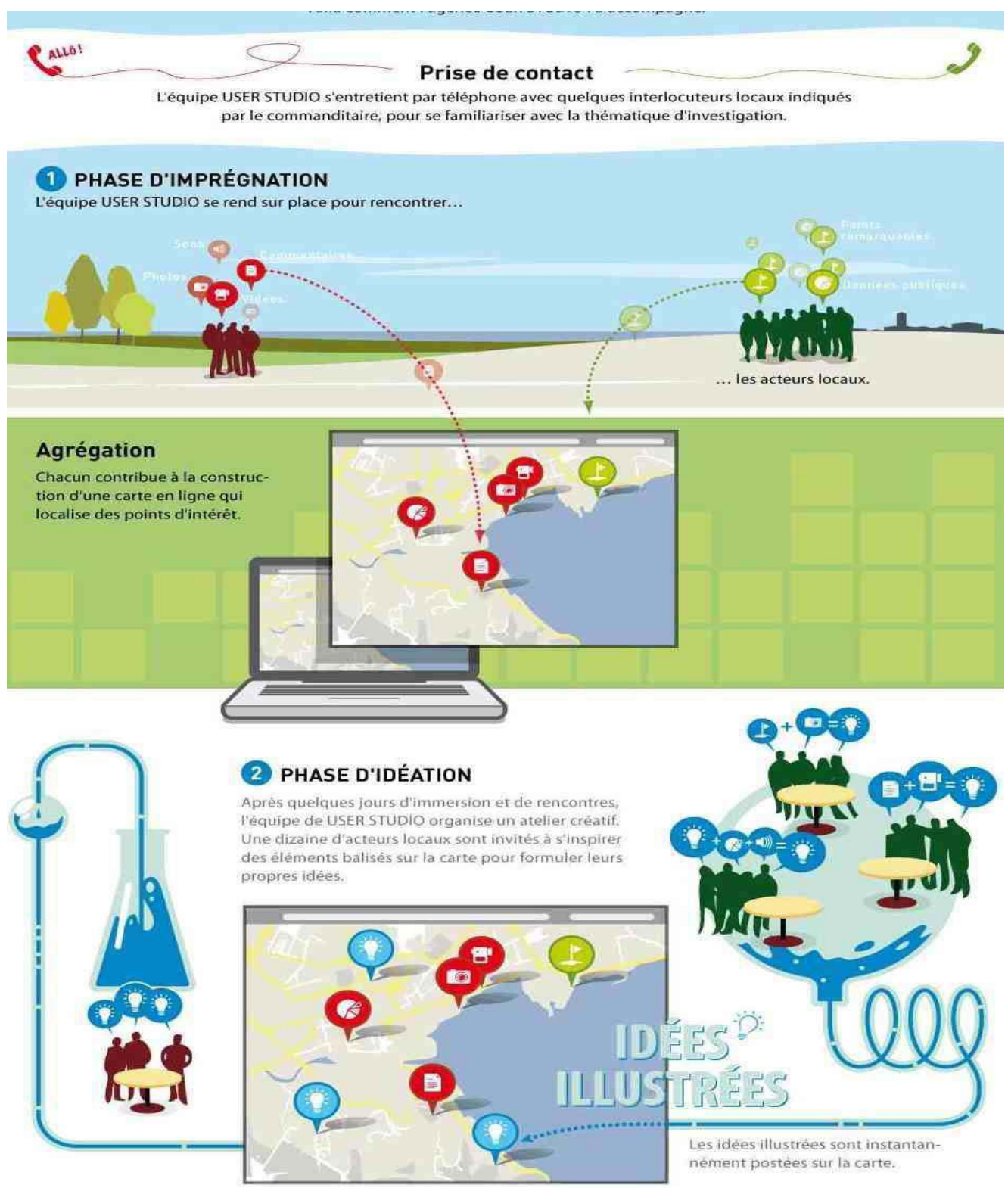


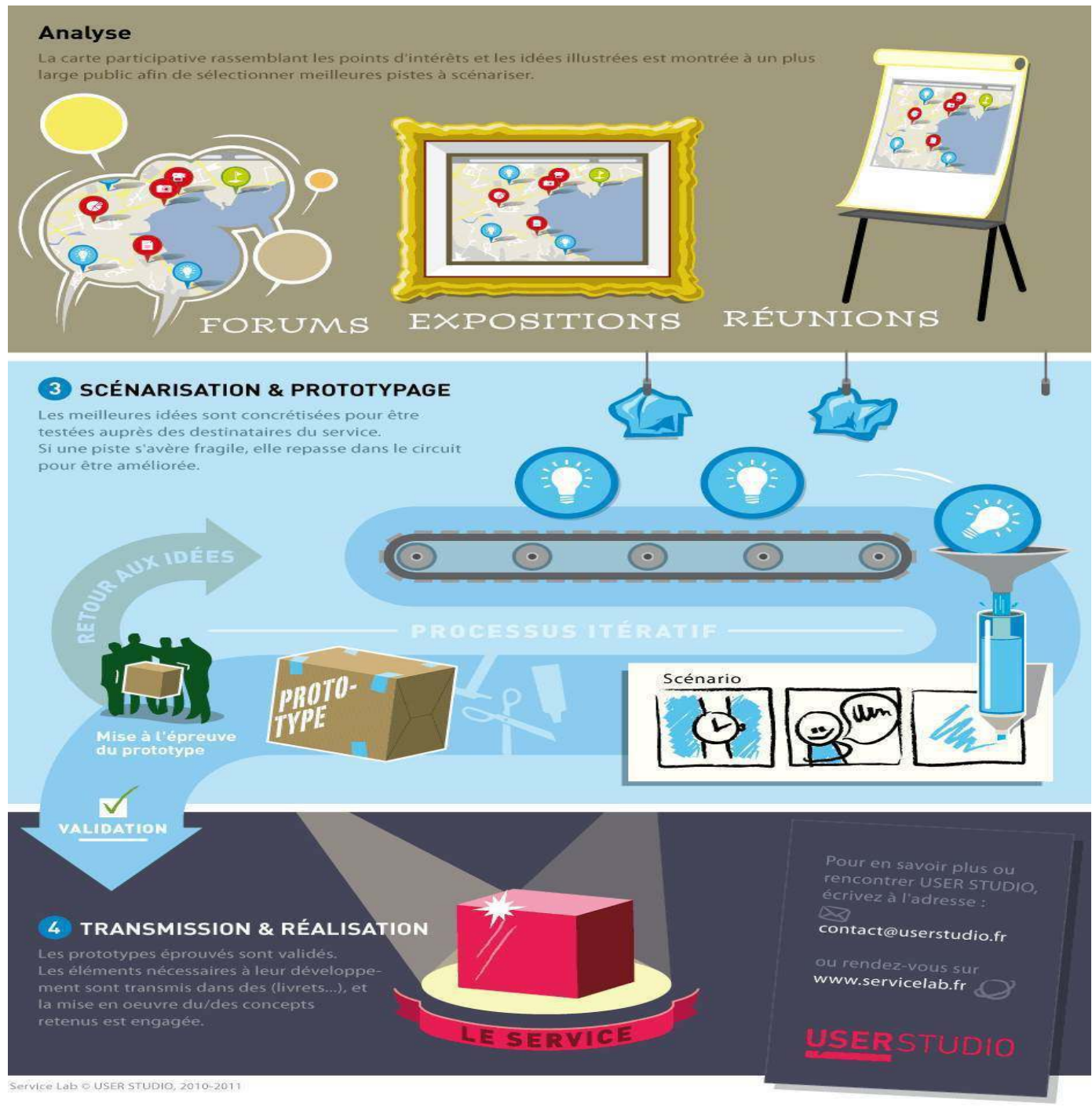

Ce procédé de séquentialisation traduit l'emprise grandissante des logiques de rentabilité sur le marché de la participation : chaque séquence permettant de proposer une multitude d'outils (outil d'information $d u$ public, outil de consultation du public, outil de dialogue) et d'assurer en prime des activités de conseils aux commanditaires. Ce travail de séquençage a ainsi contribué à la mise en œuvre de gabarits, de dispositifs « clés en main » ou encore de kit, répondant aux contraintes du marché et rendant possible une rentabilité des entrepreneurs de la démocratie participative : " L'appellation « boîte à outils » ou « kit » est très courante dans 
les sciences de gestion et désigne un manuel, un support de formation, d'évaluation ou une base de données à usage pratique destinée à orienter un usager dans sa pratique professionnelle. $»^{14}$

Ce procédé traduit également l'imprégnation grandissante des techniques de communication et managériales dans les instances participatives. Le séquençage de la participation nécessairement adossé à l'existence d'un outillage toujours plus complexe, permet en effet à des consultants en mal de diversification d'élargir leurs services aux enjeux de la participation, en adaptant à moindres frais leurs "boîtes à outils " traditionnelles. On y retrouve ainsi les codes $\mathrm{du}$ conseil en management : l'importance de la phase contact (le prospect), la même poursuite d'objectifs d'une ambition excessive par rapport aux moyens alloués, la présence du triptyque (client, consultant, public cible) ${ }^{15}$. Les différents professionnels investis sur le créneau de la participation adaptent ce séquençage à leurs prestations. Les cabinets de conseils réinvestissent, par exemple, dans le domaine de la concertation les études de contexte, les audits (qui sont présentés comme des étapes essentielles de tout projet de concertation). Ils ont en effet bien compris « le souci des porteurs de projet de maîtriser les sources d'incertitudes et donc de disposer d'informations quant aux actions pouvant être entreprises et aux arguments pouvant survenir avant même que le débat ne soit ouvert », comme le souligne Sandrine Rui ${ }^{16}$. Les agences de communication proposent, elles, que les débats soient les plus larges possibles et les plus relayés au niveau médiatique pour garantir une certaine visibilité à leurs prestations dans l'optique de susciter de nouvelles demandes. Pour s'assurer une rentabilité, les prestataires insistent alors sur la multiplication des outils communicationnels (publication de journaux, expositions, plaquettes, opérations événementielles).

Enfin, cette séquentialisation est également induite par le projet participatif lui-même : comme la pédagogie, ce projet requiert de disposer d'espaces de reformulation dont les formats sont anticipés (écrit/oral/synthèse) brainstorming/rapport final, etc.) selon une logique temporelle de progression. Le découpage en étapes vient naturellement représenter ce procès, au sens propre de représentation visuelle et formelle. Celui-ci est constitué par des genres de textes et de modalités d'expression auxquels est dévolue la capacité à former les individus autant qu'à faciliter la participation. Dit autrement, le séquençage participatif est justifié comme le séquençage pédagogique par la progression cognitive qu'il rendrait possible. Cette dimension du séquençage des procédures permet de pointer un aspect : la standardisation provient autant de facteurs économiques (rationalisation de la gestion de projet) que de croyances dans les outils comme des « technologies intellectuelles » efficaces.

Ainsi, toute démarche participative est aujourd'hui nécessairement devenue programmatique et découpée en étapes incontournables à suivre, sur laquelle les consultants proposent leurs méthodes etdéposentparfois des copyrights. Comme le suggère habilement l'en-tête du site de « Service Lab » exposé précédemment, les collectivités territoriales seront accompagnées de « $\mathrm{A} » \mathrm{à} 《 \mathrm{Z} »$, selon des étapes que seul le 
professionnel maitrise, obligeant dans une certaine mesure le commanditaire à avoir recours au même prestataire. Ces derniers se proposant d'être à la fois ceux qui l'animent, le conçoivent, l'évaluent et en assurent le suivi.

\section{Les aménagements sémantiques du discours sur les procédures}

Outre ce séquençage des procédures, la standardisation des prestations peut également s'expliquer par le minutieux travail d'ajustement sémantique qui s'opère sur le secteur de la participation. Tout d'abord, l'importation de techniques issues du conseil en management, de la communication ne s'est, en effet, pas faite sans opérer certains aménagements sémantiques censés attester du caractère participatif des prestations proposées. Ces glissements terminologiques renvoient d'ailleurs, à bien des égards, à ceux déjà analysés dans le secteur de l'humanitaire ${ }^{17}$, secteur confronté à l'imprégnation grandissante des méthodes utilisées dans la vente par correspondance pour gérer et dynamiser les donations, le fundraising. Dans des secteurs où le principe wébérien de rationalité en valeurs prédomine, l'importation des pratiques managériales nécessite ainsi certains ajustements.

Ces reformulations sont loin d'être propres au milieu du conseil et de la communication plus récemment positionnés sur le champ de la participation. Chaque univers professionnel semble importer son vocabulaire propre en l'adossant au lexique de la participation. C'est le cas des urbanistes et architectes, mais aussi des entrants plus récents sur le marché de la participation. On pense notamment à l'arrivée massive depuis ces dernières années des graphistes et des communicants. L'exemple étudié dans l'encadré 1 est, à ce titre, à nouveau exemplaire de ces aménagements sémantiques : les acteurs mobilisent un lexique qui contourne le politique en renvoyant au secteur de la communication et du design d'interface (ce que renforce également la mise en forme graphique dans le support de communication). Pourvoyeuse de contenus pour des supports numériques, User studio conceptrice de l'application " service lab » est représentative de l'arrivée de nouveaux acteurs situés à mi-chemin entre design numérique, technologie et démocratie participative. Les acteurs de cette agence réalisent aussi bien « un accompagnement des collectivités dans la création de services aux habitants » qu'un travail de « traitement de l'information par une approche design de la visualisation de données » pour un service bancaire. Dans cet exemple, deux champs lexicaux s'entrechoquent : celui de la conception de projet numérique (agrégation, scénarisation, prototypage, validation, réalisation) et celui du débat public lié à l'action publique (acteurs locaux, idéation, idées illustrées, analyse, forums, expositions, réunions, processus itératif, transmission, service).

Plus largement, cette attention linguistique s'objective aussi dans la capacité des professionnels investis sur le champ de la participation d'adapter constamment leur lexique au champ lexical de la participation. Dans ce travail sémantique, l'adaptation s'avère souvent peu coûteuse. Elle peut consister en l'habillage des prestations proposées par un 
vocabulaire participatif (« audit participatif »); elle peut également relever de l'appropriation par les consultants des appellations désormais labellisées : budget participatif, petits-déjeuners participatifs, etc. Dans le domaine de la concertation sur les questions d'aménagement urbain, il n'est alors pas rare de retrouver des dispositifs qui portent le même nom mais qui au final reposent sur des dynamiques très différentes. On pense notamment à l'appellation " atelier de travaux urbains » utilisée très fréquemment dans le milieu de la participation pour définir des dispositifs parfois sans commune mesure. Elle est généralement employée en référence à l'Atelier Populaire d'Urbanisme de l'Alma-Gare pour qualifier des dispositifs au sein desquels les habitants sont invités au même titre que les élus et les techniciens à donner leur avis et formuler des propositions concernant des projets d'aménagement en cours sur un quartier ou une commune ${ }^{18}$. Les « ateliers de travaux urbains » modernes semblent recouvrir des réalités diverses parfois très éloignées de cet idéal original. Comme le suggèrent des observations réalisées dans le Nord $^{19}$, ils correspondent sur le terrain à des pratiques bien distinctes dont le flou entretenu entre elles n'est pas le fruit du hasard, "le moins laissant croire au plus $»^{20}$. Face à l'explosion de ce lexique participatif indigène, il devient de plus en plus difficile de distinguer les prestations les unes des autres, renforçant par là même l'impression d'homogénéisation du milieu des professionnels de la participation.

Le processus de professionnalisation et de marchandisation de la participation semble ainsi avoir conduit à une dynamique de standardisation des pratiques des professionnels. Il s'est opéré un glissement au fil duquel la participation définie comme projet et comme expérience collective a été de plus en plus confondue avec les prestations proposées par les divers professionnels œuvrant sur ce secteur. Ce processus atteint une quintessence dans la production de « kits » participatifs reproductibles et transférables tels quels sur n'importe quel territoire et pour le compte de n'importe quelle collectivité. Notons que cette évolution, loin de toucher les seuls derniers arrivants sur le marché, a également affecté des structures plus anciennes et traditionnellement plus attachées à défendre une conception avant tout militante de la participation ${ }^{21}$. Or cette dynamique n'a pas été sans effet sur l'offre de participation.

\section{Développement de l'ingénierie participative et « monstration » de la participation}

Le développement de l'ingénierie participative et l'apparition de « kits » participatifs a produit de nombreux effets sur le marché de la participation et plus largement sur les politiques participatives que nous ne pouvons explorer ici de manière exhaustive tant le champ des questionnements est vaste. On pense notamment aux dynamiques de conversion et de reconversion des professionnels de la participation, l'arrivée de nouveaux profils sur ce créneau (communication, design de l'interface) semblant étroitement être corrélée avec le départ de certains (issus notamment de la politique de la ville). De même, on pense aux effets que cette standardisation du milieu a pu avoir sur la production de la commande publique participative, ou bien au possible contournement 
de la question des effets de la participation sur la décision publique qu'offre aujourd'hui la focalisation sur l'outillage. On se concentrera ici sur ce que cette dynamique de mise en « kit» de la participation a fait à la participation.

Autrement dit, nous souhaiterions montrer en quoi, d'une part, ces « kits » participatifs ont contribué à réduire la participation à la prestation proposée. D'autre part, nous souhaitons mettre en relief comment, en réduisant la participation à l'outil, ces « kits » illustrent une tendance observable sur le marché de la participation, certes de manière caricaturale : la part plus importante des professionnels consacrée à faire la « monstration » de la participation, c'est-à-dire à œuvrer à authentifier que la participation a eu lieu, renforçant encore davantage la réduction de la participation aux outils et prestations proposées.

\section{La confusion des genres : l'outillage est la participation}

La centralité accordée à l'ingénierie dépasse aujourd'hui les simples discours. Aussi, si la participation est devenue une prestation, parfois présentée sous la forme de " kit », il semble bien que la prestation finisse par symboliser dorénavant la participation. Cette cristallisation sur les outils et les compétences nécessaires pour les maîtriser permettant notamment de justifier par elle-même l'existence des professionnels sur le secteur de la participation. Il existe ainsi une confusion des genres entre dispositif, outillage et participation. Dans cette perspective, ce ne sont plus tant les propriétés singulières des concepteurs qui comptent mais le produit en tant que tel, autrement dit le mode d'emploi participatif qui va être conçu et vendu par les prestataires. On observe ainsi un processus d'effacement des acteurs derrière les outils participatifs proposés et dont l'essentiel du travail peut parfois se réduire à la production du « kit ». Or, la centralité désormais accordée aux outils est loin d'être sans effet. Le dispositif, en devenant l'objet central du discours sur la participation et des pratiques des professionnels, finit par entretenir l'illusion que l'outil peut se suffire à lui-même, et qu'il est alors en mesure d'incarner à lui seul la participation ${ }^{22}$. C'est l'hypothèse que nous faisons.

L'exemple de l'encadré 1 décrit d'ailleurs parfaitement ce mécanisme. La métaphore de la chaîne de traitement des « idées » (icône de l'ampoule, tapis roulant et automatisation etc.) est particulièrement efficace puisqu'elle définit un processus parfaitement linéaire et produisant forcément quelque chose. Une fois engagés, les différents protagonistes ne peuvent reculer et sont « pris » dans le dispositif. La mécanisation symbolique ainsi illustrée décrit une opérativité immanente du dispositif et sa capacité à s'effectuer dans sa totalité. D'une certaine manière, on peut dire que les professionnels rendent ostentatoire la nature de « technologie intellectuelle » des outils participatifs qu'ils mobilisent et prennent particulièrement appui sur ces outils pour donner à voir la participation, bien souvent réduite à une mise en scène de l'interaction (la table ronde, la réunion créative, le brainstorming, etc.). Le recours à des sites web reproduisant les caractéristiques éditoriales et logicielles des sites de réseaux sociaux est un exemple du brouillage des frontières entre ce qui relèverait du débat et ce qui relève des modalités 
fonctionnelles de participation (cliquer, publier, etc.), dans des normes éditoriales contraintes par la nature même du texte numérique (par exemple, la publication antéchronologique sur un blog) $)^{23}$. L'outillage participatif finit ainsi par donner l'illusion d'englober toutes les interactions nécessaires à la mise en œuvre de la démarche participative. Dans l'exemple 1, le processus comprend des formats variés dont la diversité et la dimension tautologique est un des arguments : des discussions interpersonnelles en face à face (tables de café, forums, réunions), des supports d'écriture et de publication numériques, des expositions etc.

Ce mécanisme de réduction de la participation à l'outil est aussi facilité par l'usage de plus en plus important du numérique dans les prestations proposées. Le statut sémiotique des formats de la participation est en effet rarement étudié dans les recherches. Or, les acteurs de la démocratie participative survalorisent parfois la qualité des dispositifs conçus en naturalisant les liens entre les caractéristiques participatives de tel ou tel outil et la capacité de l'outil à produire de la participation.

\section{Quand authentifier la participation devient la clé de voûte de l'ingénierie participative}

Si l'apparition de « kits » participatifs tend ainsi à faire du professionnel un élément de l'outillage, elle semble aussi considérablement orienter l'activité de ces acteurs sur la seule optique d'une « monstration » de la participation, accélérant encore davantage la réduction de la participation à la seule prestation proposée. À ce titre, l'univers des professionnels de la participation semble avoir sensiblement évolué depuis nos recherches doctorales ${ }^{24}$ célébrant l'arrivée sur le marché d'une nébuleuse de prestataires centrés sur la production d'outils participatifs vendus clés en main, comme le propose User studio. Les outils présentés intègrent alors dans leur fonctionnement comme dans leur design tous les moyens nécessaires aux collectivités territoriales de faire la preuve de la participation.

Si les méthodes diffèrent d'un consultant à l'autre pour authentifier la participation, là encore derrière l'hétérogénéité des pratiques, il est possible de dégager des caractéristiques communes. Pour les commanditaires de l'action publique, la mise en œuvre de procédures de participation suppose, en effet, d'en laisser une trace, condition sine qua non de l'évaluation de la démarche. Selon les enjeux et les acteurs, l'enjeu de transparence va se traduire par la publication d'un site web (forum, blog etc.), de journaux, etc. La surenchère des supports numériques ou d'espaces de débat électronique comme dispositifs adossés aux démarches participatives permet ainsi de donner assez facilement des réponses automatisées à l'authentification que le débat a eu lieu. Les sites web vont alors servir de support d'évaluation pour les experts. Les documents numériques répondent à la double fonction de rendre possible le débat et d'en archiver la procédure. De fait, cette quête à l'authentification de la participation est autant le produit des professionnels eux-mêmes que des commanditaires de plus en plus friands d'évaluation des dispositifs préformatés. Dans ce processus, la communauté scientifique joue bien évidemment un rôle non négligeable, en témoigne la présence de garant chargé 
d'évaluer la qualité des débats s'est aujourd'hui largement institutionnalisée dans les démarches participatives.

Les outils proposés par ces nouveaux types de prestataires jouent ainsi sur l'ambiguïté entre les fonctions : archiver, en même temps qu'on agit. Loin d'être anodine, cette remarque interroge directement le statut communicationnel des documents et plus largement le statut des participants, amplement confondus en raison de la nature spécifique du texte informatisé qui marie les espaces de la pratique, de la mémoire et de l'inscription ${ }^{25}$. À quoi servent ces outils de lecture et d'écriture dans le contexte participatif? À produire du débat ou à montrer que quelque chose se passe, ou s'est passé ? Ainsi, l'authentification est sémiotisée par le biais de la combinatoire automatisée d'un site web : en effet, l'organisation en bases de données permet de mobiliser selon différentes logiques énonciatives les contenus qui alimentent le site. Par exemple, les contributions écrites des participants pourront apparaître selon plusieurs représentations documentaires : nuage de tags, dernier contributeur, contributeur le plus actif, sujet le plus commenté, etc. L'utilisation des supports numériques de la participation (reposant principalement sur les plates-formes de type Content Management System) engendrent un cercle vicieux : leur statut communicationnel de « vitrine » du débat nécessite leur actualisation et renforce la nécessité de laisser des traces de l'activité ; en cela, on peut dire que les formes numériques de publicisation du débat « instrumentalisent » les participants potentiels.

$\mathrm{Au}$ risque de passer sous silence les logiques de conflits et les stratégies de démarcation aujourd'hui à l'œuvre sur le marché de la participation, cette contribution exagère volontairement les logiques d'homogénéisation et de standardisation de l'ingénierie participative. En se centrant sur les multiples dimensions de l'ingénierie participative, englobant à la fois les acteurs et leurs propriétés tout comme les outils qu'ils produisent, nous avons, en effet, souhaité éclairer un angle de la participation encore trop peu exploré, celui de la part ingénierique de la participation. Si l'existence de ces « kits» participatifs ne caractérise pas encore l'ensemble des prestations et des pratiques proposées sur le marché de la participation, on ne peut les occulter en raison des effets qu'ils produisent sur la nébuleuse de professionnels aujourd'hui investis dans ce créneau. Les pratiques s'homogénéisent et les outils se standardisent. L'intérêt du double positionnement sociologique et sémiologique permet également de montrer que les formats choisis pour élaborer ces « kits » produisent eux-mêmes des effets : ils renforcent par exemple la réduction de la participation à l'outil ; ils exacerbent la part consacrée par les professionnels à penser en amont les moyens d'assurer la « monstration » de la participation.

Cette analyse des « kits » participatifs laisse bien évidemment en suspens la question des usages de ces outils participatifs par les participants, et notamment celle des possibilités de détournement, de contournement et d'appropriation de ces mêmes outils. Néanmoins, l'entrée par l'ingénierie laisse émerger plusieurs interrogations fondamentales. Notre approche questionne, par exemple, la place des spécialistes et de l'expertise dans le champ de 
la participation. Dit autrement, la participation ne finirait-elle pas devenir une affaire de spécialistes ? Tout d'abord, la « technicité intellectuelle » et culturelle des outils utilisés aujourd'hui sur le marché de la participation invite à considérer toutes les dimensions de cette question. L'application numérique développée par User Studio pour représenter les « rêves des habitants » dans une « carte de la participation » interroge, en effet, la capacité des acteurs à se saisir de ces représentations du débat et de l'espace particulièrement stylées et sélectives, et dont le lissage graphique et le cadrage discursif laissent peu de place au bricolage et à des interprétations contrastées. Deuxièmement, si le terme de « kits » participatifs nous semble fécond pour décrire la production de prestation livrée clés en main, transférable et reproductible à l'infini, il n'est peut-être pas totalement adéquat si l'on considère que la caractéristique principale des « kits » tient également dans la possibilité qu'à l'acheteur de monter lui-même les éléments à la manière d'un meuble Ikéa. Nous l'avons montré, la technicité des outils nécessite aussi parfois la maîtrise de compétences que seuls les professionnels détiennent et qui vont conditionner la nécessité de faire appel à une assistance. Cela s'avère encore plus marqué dans le cas de prestations où les professionnels proposent déjà les services d'évaluation, d'animation et de suivi des dispositifs. Cette dynamique explique en quoi aujourd'hui ce type de prestataires arrive à gagner de plus en plus de marchés publics sur le créneau de la participation. Enfin, on peut aussi se demander dans quelle mesure ces " kits » participatifs ne sont finalement pas le reflet le plus visible d'une confiscation symbolique de la participation au profit des spécialistes. Ces évolutions laissent craindre l'avènement d'un procéduralisme contrôlé par un petit groupe de professionnels et attestent en tout cas d'une division du travail participatif au sein même du marché de la participation ${ }^{26}$. 
$\mathrm{N} \cdot \mathrm{O} \cdot \mathrm{T} \cdot \mathrm{E} \cdot \mathrm{S}$

1. M. Nonjon, Quand la démocratie se professionnalise. Enquête sur les experts de la participation, thèse de doctorat de science politique, Lille, 2006 ; G. Gourgues, Le consensus participatif. Les politiques de la démocratie dans quatre régions françaises, Thèse de science politique, IEP Grenoble, 2010.

2. A. Mazeaud, La fabrique de l'alternance. La démocratie participative dans la recomposition $d u$ territoire régional (Poitou-Charentes 2004-2010), Thèse de doctorat en science politique, Université de La Rochelle, 2010.

3. Ces "Rencontres nationales des professionnels de la démocratie participative" ont été organisées par le conseil Général du Val-de-Marne à Creteil le 17 et 18 novembre 2011.

4. Extrait du discours introductif prononcé par Didier Guillaume, vice-président en charge de la démocratie participative et de la vie associative du $\mathrm{Cg}$ du Valde-Marne lors de la première journée des rencontres nationales des professionnels de la participation.

5. On se concentrera pour l'article sur une illustration emblématique de ce phénomène : il s'agit de l'application en ligne « Service Lab » conçue par User Studio, une agence d'innovation par le design des services et qui était présente lors des rencontres des professionnels de la participation organisées par le Val-de-Marne parmi les prestataires invités.

6. C. Tardy, Y. Jeanneret, J. Hamard, « L'empreinte sociale d'un outil d'écriture : PowerPoint chez les consultants », in C. Tardy, Y. Jeanneret (dir.) L'écriture des médias informatisés : espaces de pratiques, Paris : Hermès Sciences-Lavoisier (Coll. « Systèmes d'information et organisations documentaires »), 2007, pp. 141-171
7. Tissot 2002, Nonjon, 2006, Nonjon, 2012 S. Tissot, Réformer les quartiers, Enquête sociologique sur une catégorie d'action publique, thèse de doctorat de sociologie, Paris, 2002 ; M. Nonjon, « De la militance à la consultance : les bureaux d'études urbaines, acteurs et reflets de la professionnalisation ", Politique et Management Public, $\mathrm{n}^{\circ}$ 29/1, 2012.

8. Cette fondation finance chaque année plusieurs petites structures encore artisanales pour la plupart sur le créneau de la participation. On pense notamment à User studio, agence d'innovation pour le design de services, Data field, support d'enquête via Smartphone, villagora.fr, plate-forme web de concertation citoyenne ou encore les clameurs, une agence proposant des campagnes scénarisées et virales sur la sonorisation géolocalisée de l'espace urbain. De plus en plus présente dans les rencontres des professionnels de la participation comme partenaire financier ou pourvoyeuse d'animations, elle entend aujourd'hui être un acteur fédératif et professionnel important sur la participation.

9. S. Labelle, La ville inscrite dans "la société de l'information ": formes d'investissement d'un objet symbolique. Thèse, Université Paris 4, GRIPIC, décembre 2007.

10. Extrait d'entretien, Paris, 2002 in M. Nonjon, op. cit., 2006.

11. On a, en effet, montré dans le cadre d'autres communications que d'une part les ressources militantes continuent d'être importantes et sollicitées par les commanditaires. D'autre part, cette vision du secteur de la participation divisée en deux mondes antagonistes, les «professionnels » versus les « militants » de la participation est largement construite par les consultants qui œuvrent aujourd'hui sur le marché de la participation. La référence à la rhétorique militante ou professionnelle de leurs pratiques relève d'un jeu extrêmement complexe qui 
dépend largement des ressources détenues par ces acteurs. Voir à ce sujet, M. Nonjon, op. cit., 2006 et 2012.

12. S. Tissot, op. cit., 2002 ; M. Nonjon, op. cit., 2006. 13. On aurait pu, par exemple, étudier l'homogénéisation des profils des consultants et leurs déplacements dans les structures œuvrant sur le marché de la participation.

14. A. Seurrat, Les médias en kit pour promouvoir la diversité. Etude de programmes européens de formation aux médias destinés à "lutter contre les discriminations " et " promouvoir la diversité 》, Thèse de doctorat en Sciences de l'information et de la communication, Université Paris - Sorbonne, Celsa, 2009, p. 44.

15. M. Villette, Sociologie du conseil en management, Repères, La découverte, 2003.

16. S. Rui, La démocratie en débat. Les citoyens face à l'action publique, Armand Colin, 2004.

17. S. Lefèvre, « Le sale boulot et les bonnes causes : institutionnalisation et légitimation du marketing direct au sein des ONG », Politix, n79, 2007, pp. 149-172. 18. M. Nonjon, op. cit., 2006.

19. M. Nonjon, «Participation des habitants et règles du jeu municipal. Une étude de cas », Cahiers lillois d'économie et de sociologie, $\mathrm{n}^{\circ} 35-36,2001$, pp. 245-266.

20. P. Lascoumes, « L'obligation d'informer et de débattre, une mise en public des données de l'action publique », in J. Gerstlé (dir.), Les effets d'information en politique, Paris, l'Harmattan, 2001, p. 311.

21. M. Nonjon, op. cit., 2012

22. J. Bonaccorsi, C. Ollivier-Yaniv, « Le «tournant délibératif» de la politique de communication de l'U.E. entre 2005 et 2009 et ses paradoxes. Le cas des Consultations Citoyennes Européennes », Communication au Colloque Médiations de l'Europe politique, Strasbourg, MISHA, 30 et 31 mai 2011.
23. J. Bonaccorsi, V. Julliard, " Dispositifs de communication numériques et nouvelles formes de médiation du politique, le cas du site web d'IdealEU » in M. Aghababaie, A. Bonjour, A. Clerc, G. Rauscher (dir.). Usages et enjeux des dispositifs de médiation, Nancy, Presses Universitaires de Nancy, 2010, pp. 65-78.

24. M. Nonjon, op. cit., 2006.

25. Y. Jeanneret, « Les harmoniques du web : espaces d'inscription et mémoire des pratiques », MEI, $\mathrm{n}^{\circ} 32$, 2010, pp. 31-41.

26. C'est d'ailleurs le programme de recherche aujourd'hui entamé par Alice Mazeaud et Magali Nonjon sur la cartographie des professionnels de la participation. Voir à ce sujet A. Mazeaud, M. Nonjon, «Enjeux et dilemme d'une cartographie des professionnels de la participation : l'apport de l'observation ethnographique d'espaces de socialisation et de professionnalisation ", communication présentée au congrès de la Société Québécoise de Science Politique, mai 2012. 


\section{$R \cdot E ́ \cdot S \cdot U \cdot M \cdot E ́$}

L'article souhaite éclairer un angle de la participation encore trop peu exploré, celui de la part ingénierique de la participation engageant des médiations trop souvent naturalisées. La posture méthodologique choisie articule approche sociologique et sémiologique des pratiques et des supports développés aujourd'hui par certains professionnels de la participation. Cette recherche s'intéresse ainsi à l'émergence d'une participation en « kit ». Elle met en relief la professionnalisation du secteur de la participation, ses dynamiques d'homogénéisation et de standardisation des pratiques, en analysant notamment le processus de naturalisation des liens entre outils et participation mis en scène par les consultants. Elle souligne également que les formats choisis pour élaborer ces " kits » produisent euxmêmes des effets : ils renforcent par exemple la réduction de la participation à l'outil; ils exacerbent la part consacrée par les professionnels à penser en amont les moyens d'assurer la « monstration » de la participation.

\begin{abstract}
The article aims to analyse a too little explored dimension of public participation: the engineering that underline the "production » of public participation, which is too often naturalized. According to a sociologic and semiotic approach, we focus on the practices and tools used by some " professionals " of participation. Our research is interested in the emergence of " sectional participation ». We highlight the consolidation of a genuine professional market, the standardization and homogenization of practices, by analyzing the process of naturalization of the links between participation and its tools led by
\end{abstract}

consultants. The formats that professional choose in order to develop these « sectional participation » are producing their own effects: for instance, they reduce participation to a tool; they drive professionals to insist on the « demonstration » and of the participatory qualities of their tools. 